\title{
Dinámicas de colaboración en las organizaciones del tercer sector de acción social en España
}

\section{Germán Jaraíz Arroyo, Auxiliadora González Portillo}

RESUMEN: La evolución y posicionamiento del tercer sector están vinculados a la morfología de sus redes propias y la conexión cívica mediante la que estas organizaciones tejen sus tramas. Este trabajo analiza la dimensión relacional del sector.

El artículo presenta una parte de los resultados del Proyecto Perspectiva Comparada del Tercer Sector (en adelante PECOTSAS), los referidos al análisis del proceso de articulación del tercer sector de acción social en España y su aporte en la generación de capitales sociales. Pretendemos identificar los principales elementos incidentes en este proceso en un grupo de siete comunidades autónomas para discernir en qué medida su evolución ha dado lugar a diferentes desarrollos autonómicos de tercer sector.

En lo metodológico, los resultados de investigación se han obtenido, por un lado del análisis bibliográfico y de fuentes secundarias (análisis descriptivo), y por otro del desarrollo de 48 entrevistas en profundidad a informantes clave (gestores, técnicos, voluntariado, stakeholders) y 14 grupos focales (análisis de las percepciones).

El trabajo concluye que, aunque el tercer sector es en esencia un actor cívico, en la configuración de sus redes han ejercido mayor influencia los aspectos de colaboración-prestación de servicios en contextos de política pública, siendo también influyentes la defensa de derechos, la producción y divulgación interna de conocimiento y el fomento de la participación voluntaria. Por último concluimos que, si bien es cierto que cada una de las comunidades analizadas posee singularidades, el condicionamiento de elementos de conjunto en el ámbito europeo y estatal ejercen una presión homogeneizadora mayor.

PALABRAS CLAVE: Tercer sector, colaboración, redes, sociedad civil.

CLAVES ECONLIT: I3, L3, N9, R5. 
Cómo citar este artículo/How to cite this article: JARAÍZ-ARROYO, G. \& GONZÁLEZ-PORTILLO, A (2021): "Dinámicas de colaboración en las organizaciones del tercer sector de acción social en España", CIRIEC-España, Revista de Economía Pública, Social y Cooperativa, 103, 141170. DOI: 10.7203/CIRIEC-E.103.21498.

Correspondencia: Germán Jaraíz Arroyo, Departamento de Trabajo Social y Servicios Sociales, Universidad Pablo de Olavide de Sevilla, gjararr@upo.es, ORCID: 0000-0001-9944-6536; Auxiliadora González Portillo, Departamento de Trabajo Social y Servicios Sociales, Universidad Pablo de Olavide de Sevilla, magonpor@upo.es, ORCID: 0000-0003-1943-6122.

ABSTRACT: The evolution and positioning of the Third Sector as an actor is linked to the nature and morphology of its networks and to the spaces of civic connection-collaboration around which Third Sector organisations weave their webs. This study analyses the relational dimension of the sector.

The article presents some of the results of the PECOTSAS project, referring to the analysis of the articulation process of the TSSA in Spain and its contribution to the generation of collective forms of social capital. We intend to identify the main elements involved in this process in a sample of seven Autonomous Communities in order to discern the extent to which their evolution has given rise to different regional developments of the Third Sector.

In methodological terms, the research results have been obtained, on the one hand, from the analysis of the literature and secondary sources (descriptive analysis), and on the other hand, from the development of 48 in-depth interviews with key informants (managers, technical staff, volunteers, stakeholders and 14 focus groups (analysis of perceptions).

The study argues that, although the Third Sector is essentially a civic actor, the configuration of its networks has been largely shaped by the aspects of collaboration and service provision in public policy contexts, with advocacy, the production and internal dissemination of knowledge and the promotion of voluntary participation also being influential. Finally, we conclude that, although it is true that each of the regions under analysis presents singularities, a set of conditioning elements at the European and state levels exert a homogenising pressure on the networks.

KEYWORDS: Third sector, collaboration, networks, civil society. 


\section{Expanded abstract}

\section{Collaboration dynamics in third sector social action organizations in Spain}

\section{Context and Objectives}

The evolution and positioning of the Third Sector as a civic actor is linked to the nature and morphology of its networks and to the spaces of connection-collaboration around which Third Sector organisations weave their webs. This study analyses the relational dimension of the sector.

The development and expansion of Third Sector organisations in Spain (hereafter referred to as TSOs) has been conditioned by the (late) process of adaptation to a model of welfare capitalism in which a market economy coexists with a state that has the capacity to guarantee and provide a part of social goods and a space that is open to civic participation. The Spanish Third Sector has evolved within this context. In this process, different initiatives and dynamics of internal collaboration (between organisations) and external collaboration (with other actors) have been generated. On the other hand, the adoption of a state model of Autonomous Communities following the democratic transition and the fact that competencies for the majority of social policies have been transferred to the regions have brought elements of territorial differentiation into the overall process.

In this study, we analyse the overall influence of the forms of collaboration adopted by Third Sector organisations, exploring the effect they have had on the formation of the sector itself, as well as their social and political impact.

The approach of the study is based on two research questions. On the one hand, we are interested in identifying the relational logics and the purposes and effects sought in the sector's collaboration processes. We start from the hypothesis that, although the dynamics of collaboration are marked by the aforementioned scenario, the effects of these collaborations among TSOs are diverse and have to do with their overall institutional positioning, with the generation of discourses that affect society and public policies, and with their role as sources of social capital and participation. On the other hand, the importance of the regional factor forces us to take a comparative look at different territorial processes. In this sense, our work raises some questions: do different logics exist with respect to the processes of collaboration in the autonomous regions, and if so, do they condition the formation of different regional welfare models?

\section{Theoretical aspects and methodology}

In relation to the theoretical aspects, it is necessary to begin by identifying the predominant conception of the Third Sector for the case study. Ángel Zurdo (2007) differentiates between two approaches or macro-conceptions: in the first, referred to as the Anglo-Saxon or non-profit 
approach, the solidarity and non-profit nature predominates above all else as the articulating factor of the entities, normally concretised in organisational terms through associations or foundations. The second approach, referred to by Zurdo as the continental approach, resorts to a broader idea of the sector, contemplating within it all forms of social economy, cooperative economy and alternative economy to traditional market logics. In the Spanish case, the identification of the sector with the non-profit approach has predominated. Within this field, a distinction is also made between organisations operating in the field of social services, known as Third Sector of Social Action Organisations (TSSAOs), and those operating in such fields as culture and the environment.

With respect to the forms of collaboration between TSSAOs, the predominant idea is that these relationships focus on the search for positioning in the context of a service market (Ruíz de Olabuénaga, 2006). However, our starting point here is a broader vision, understanding the sector as an actor that generates different types of responses to collective problems in a changing environment (Astley \& Fombrun, 1983), thus contemplating other sources of collaboration in terms of advocacy functions (Beaton, Macindoe \& Wang, 2021) as well as social participation and social capital (Saz-Gil et al., 2016).

The last of the theoretical elements guiding the research is related to the types of collaboration brought into play by the TSSAOs. To this end, we delve into the contributions of various authors such as Agranoff and McGuire (2003), who differentiate between collaboration and partnership, the former taking a more hierarchical form, while the latter tends to be more mutualistic; or Iglesias and Carrera (2013), who distinguish between tactical and strategic collaboration, and point out three types of the latter: strategic alliances around a common objective, institutional integrations and networks or platforms.

Methodologically, the research results were obtained, in their descriptive part, from the analysis of the literature and of secondary sources (reports of the different networks, content analysis of web pages, corporate information). For the analysis of perceptions, a total of $48 \mathrm{in}$ depth interviews were conducted with key informants (managers, technical staff, volunteers, stakeholders) and 14 focus groups (two in each region). Qualified sampling procedures were used in both cases. For the study of similarities and differences between regions, we selected seven cases based on significance criteria: Andalusia, Castile and Leon, Catalonia, Community of Madrid, Community of Valencia, Basque Country and Region of Murcia. The implementation of both methods was similar in each region.

\section{Results}

As a first contribution in the results section, the research shows how the Spanish Third Sector has developed a specific relational architecture in a relatively short period of time. 


\section{Figure 1. TSSAOs cross-linking process}

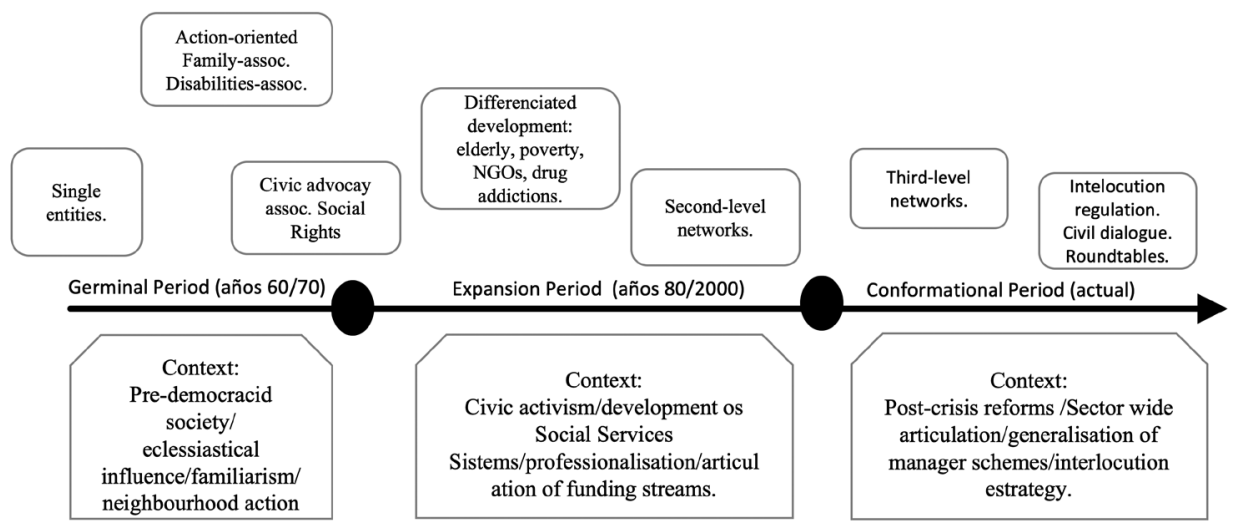

Source: Own elaboration.

In this formative process, the initial priority for TSSA0s has been their grouping into second-level networks or platforms, predominantly of a thematic nature. This thematization of the networks has had a double effect: on the one hand, it has consolidated and improved the visibility of different areas of intervention (disability, drug addiction, the fight against poverty, development cooperation, etc.), but at the same time it has led to the differentiation and dispersion of the sector. Recently, especially after the 2008 crisis, the TSSAOs have consolidated a broad third-level collaborative space, which has developed rapidly both at the national level and in most regions. This new phase has facilitated a greater concentration of the sector around common interests, essentially based on the search for a space of its own and differentiated from other social actors (trade unions, social economy, social movements...), although it is a relatively recent process.

In the informants' testimonies, there is a predominance of positive views on the achievement of the TSSAOs in articulating themselves as a unique and differentiated actor with respect to other social actors (social economy, trade union movement, etc.), although there is also a view among a large number of informants that this achievement is insufficient, advocating a refocusing of this strategy towards an internal enrichment of the sector linked to: 1) its positioning in spaces of social innovation at the local level, 2) the capacity to achieve compatibility in the adoption of its own position, and 3) its connection with other social actors.

The second of the aspects to be investigated has had to do with the existence or not of differentiated relational models between regions. In this sense, we have been able to identify, firstly, that the origins of the sector were influenced by the role of the Church and that of families (especially relevant in the field of disability), as promoters and drivers of the first layer of TSSAOs as well as of collaboration processes, across all the territories. Links with the civic-neighbourhood movement have also been common, albeit in different forms. A second 
set of conditioning factors are regionally differentiated, with a greater weight in: Catalonia (associative tradition...), the Basque Country (community culture and close relationship with the administration) and Madrid (connection with advocacy neighbourhood movements). We have termed the third type of conditioning factors contextual factors, which refer to the global and European trend towards convergence in the sector due to the generalisation of formulas for the co-production or co-creation of services among different actors.

\section{Contributions}

By bringing the three types of factors into a dialogue, we can conclude that there are territorial differences in the development of collaboration strategies: some have developed more of a civic-participatory culture, others have developed open governance and coordination with the public sphere, while still others have fostered a dense relational fabric and the formation of interest groups. These differences give specific identities to different communities; however, when considered as a whole, they do not form differentiated models of collaboration, but rather, there is currently a predominance of dynamics of homologation in which, in addition to the context, the existence of a federal relational culture that has facilitated the permeability of initiatives between territories has also been influential. This tendency towards convergence in collaboration processes has also been influenced by the permeability and capacity to transfer initiatives between territories. 


\section{Introducción}

El desarrollo y expansión de las organizaciones del tercer sector en España (en adelante 0TS), ha venido condicionado por el propio proceso de adaptación (tardía) a un esquema de capitalismo del bienestar en el que conviven: una economía de mercado, un Estado con capacidad garantista-prestadora de una parte de bienes sociales y con un espacio abierto a la participación cívica. El tercer sector español ha evolucionado en este escenario (González-Portillo \& Jaraíz, 2017). En este proceso se han ido generando las diferentes iniciativas y dinámicas de colaboración interna (entre organizaciones) y externa (con otros actores). De otro lado, la adopción tras la transición democrática de un modelo de Estado Autonómico y el hecho de que la mayoría de políticas sociales hayan sido transferidas a las Comunidades Autónomas ha incorporado al proceso elementos de diferenciación territorial.

En el trabajo analizamos el efecto de conjunto de las formas de colaboración adoptadas por las organizaciones del tercer sector, indagando sobre el impacto que las mismas han tenido en la formación del propio sector, así como la incidencia social y política.

El planteamiento de investigación se apoya sobre dos cuestiones de estudio. Por un lado, nos interesa identificar las lógicas relacionales y los propósitos y efectos buscados en los procesos de colaboración del sector. Partimos aquí de la hipótesis de que, si bien las dinámicas de colaboración vienen marcadas por el escenario ya comentado, los efectos de estas colaboraciones entre OTS son diversos y tienen que ver con factores como: su posicionamiento corporativo de conjunto, la generación de discurso incidente en la sociedad y en las políticas públicas, y con su papel como fuentes de capital social y participación. Por otro lado, la importancia del factor autonómico nos obliga a realizar una mirada comparada a diferentes procesos regionales, en este sentido nuestro trabajo plantea algunas preguntas: ¿existen diferentes lógicas respecto a los procesos de colaboración en las autonomías?, de existir ¿condicionan las mismas la formación de modelos autonómicos diferenciados?

\section{Encuadre teórico}

\subsection{Concepciones sobre el tercer sector}

La inexistencia de una concepción más o menos generalizada sobre el espacio analítico que ocupa el tercer sector es, como plantea Billis (1992), expresión del carácter poco uniforme de este tipo de organizaciones. A menudo esto se resuelve conceptualizando a estas entidades desde una mirada a extramuros, explicando al sector más por lo que no es, que por lo que es; destacan en esta línea denominaciones de uso habitual como la de Organizaciones No Gubernamentales u Organizaciones No Lucrativas. Otras aproximaciones explican al sector por características que, siendo constitutivas del mismo, tampoco lo definen de modo completo, enfatizando aspectos como el aporte del voluntariado, o su naturaleza asociativa, características ambas que ostentan buena parte de las entidades, pero no todo el sector. 
La literatura científica más reciente encuadra al tercer sector en el ámbito de las organizaciones híbridas (Jäger \& Schröder, 2014; Espósito et al, 2021). Este tipo de entidades se consolidan en las últimas décadas en el marco de la denominada Nueva Gestión Pública, abarcando por un lado las empresas públicas o participadas por el Estado y, por otro, las empresas sociales y cooperativas (Secinaro et al, 2019). Entendemos sin embargo que este territorio de lo híbrido sigue siendo demasiado difuso y renuncia a una dimensión explicativa esencial: el carácter estrictamente cívico de estas organizaciones. Este hecho trasciende la lógica dual público-privado para establecer un tercer nivel dotado de sentido propio, la producción de bienes comunes desde una esfera de interés colectivo (Rendueles, 2017).

En línea de adscripción del sector a los aspectos corporativos Ángel Zurdo (2007) diferencia dos enfoques o macro-concepciones que nos ayudan para la aproximación al objeto específico de estudio de este trabajo. El primero es denominado enfoque anglosajón o non profit, en el que predomina, sobre todo lo demás, la naturaleza solidaria y no lucrativa como factor articulador interno de las entidades, normalmente concretada en lo organizativo mediante asociaciones 0 fundaciones. En el análisis desde esta concepción son referente los trabajos de Salamon y Anheier $(1987,2001)$. En el ámbito local se inspiran en este enfoque autores como Rodríguez Cabrero (2020); Marbán, Pérez \& Rodríguez (2020); Ruíz de Olabuénaga (2006); Cabra de Luna (2016), por citar algunos. El segundo tipo, denominado por Zurdo como enfoque continental, recurre a una idea más amplia de sector, apoyándose como elemento central en la opción por formas de economía de carácter social o economía alternativa a las lógicas tradicionales de mercado (Monzón, 2009). En este segundo enfoque tienen cabida, además de los formatos asociativos anteriores, una gran diversidad de figuras como las cooperativas, las empresas de inserción o los centros especiales de empleo.

La diferenciación entre entidades sociales no lucrativas de naturaleza solidaria y organizaciones de economía social y cooperativa está muy presente en el panorama español. Esta diferenciación marca en origen el asunto que aquí nos ocupa, el desarrollo de las dinámicas de colaboración entre las organizaciones, conformando espacios de interés diferenciado. Las entidades más sensibles a la cultura non profit han provocado diferentes alianzas con entidades guiadas por criterios referidos principalmente al ámbito de intervención social (cooperación al desarrollo, drogodependencias, medio ambiente, pobreza y desigualdad...), produciendo estructuras de red de segundo o de tercer nivel ${ }^{1}$. Paralelamente, las organizaciones más asentadas sobre su identidad económico-corporativa se han agrupado principalmente en torno a redes más vocacionadas a la promoción y protección del modelo de gestión social de la organización ${ }^{2}$, dejando en segundo lugar las cuestiones relativas a la misión específica.

1. Las entidades de segundo nivel se caracterizan por su agrupación en base a colectivos o problemáticas comunes, un ejemplo de ello será la European Antipoverty Network (EAPN), que agrupa a entidades dedicadas a la inclusión social y la lucha contra la pobreza. Las organizaciones consideradas de tercer nivel son aquellas que agrupan a organizaciones de primer y segundo nivel, la entidad referente en la actualidad en el ámbito español sería la Plataforma del Tercer Sector.

2. La red referente en este sentido es la Confederación Empresarial Española de Economía Social (CEPES), donde tiene un gran peso el sector cooperativo. Existen también otras redes en este ámbito más enfocadas a planteamientos de economía alternativa como por ejemplo la Red de Redes de Economía Alternativa y Solidaria (REAS). 
A pesar de que esta doble estrategia reticularizadora es clara en el panorama local, existe al mismo tiempo una notable permeabilidad entre ambos espacios (Crespo, 2013). Resulta frecuente encontrar entidades de carácter non profit que desde sus programas de intervención tutelan o apoyan organizaciones de economía social, como empresas de inserción, y participan en redes de colaboración del ámbito estricto de la economía social.

El trabajo de investigación desarrollado desde el Proyecto PECOTSAS se centra en la situación de las redes de entidades de lógica non profit en las diferentes comunidades autónomas. Acotando incluso más el objeto de estudio, hemos trabajado de modo específico sobre lo que autores como Marbán, Pérez \& Rodríguez (2020) han denominado organizaciones del tercer sector de acción social (en adelante OTSAS), ámbito referido a esa parte del sector que une a la racionalidad non profit, la vocación por la participación en el espacio de la desigualdad y la inclusión social. Nos referimos aquí a las organizaciones que concentran sus programas y servicios en torno a lo que, con sentido amplio, Casado \& Fantova (2007) han denominado la rama de los servicios sociales, esta elección deja fuera del foco el comportamiento de entidades en otros ámbitos (como el cultural, el medioambiental, etc.).

\subsection{Sobre la idea de colaboración en el sector}

El tercer sector adquiere consistencia como actor político en la medida en que es capaz de adquirir peso cívico, social y económico, para ello son esenciales los procesos de colaboración interna y externa (Jaraíz, 2015). Sin embargo, el interés por el estudio de esta cuestión ha sido poco frecuente, especialmente si se compara con ámbitos como el empresarial o el de las administraciones públicas. A pesar de ello, pueden encontrarse en nuestro contexto algunos trabajos de interés sobre aspectos de colaboración entre entidades (Vélez, 2007; Nieto, 2009; Carreras \& Iglesias, 2013).

A la escasez de aportes hay que unir también que la mayoría de investigaciones aplican miradas de corte gerencial (Alexander, 2000) o análisis dedicados al estudio de los efectos de la mercantilización del sector (Eikenberry \& Kluver, 2004). La tendencia en este sentido ha sido entender que el sustento relacional del sector se concentra, fundamentalmente, en la búsqueda de ventajas competitivas que no podían ser logradas por separado (Jarillo, 1998), partiendo de la base de que estas entidades tienen como único nicho institucional posible la cobertura del espacio vacío entre sociedades mercantiles y administraciones públicas (Evers, 1993).

Aun siendo cierto que uno de los aspectos que han ejercido de lanzadera de determinadas dinámicas de cooperación es la entrada del tercer sector en el mercado de los servicios sociales (Ruíz de Olabuénaga, 2006), limitar la colaboración entre entidades a cuestiones de posicionamiento respecto la provisión de servicios es aplicar un reduccionismo que deja fuera aspectos esenciales en la identidad del sector. Las OTSAS combinan habitualmente la labor prestadora con funciones de advocacy (Beaton, Macindoe \& Wang, 2021), con la promoción de la participación y el desarrollo de capital social (Saz-Gil et al, 2016), fruto de su vinculación organizativa e identitaria con lo socio-cívico (característica diferenciadora respecto a los ámbitos mercantil y público) (Jaraíz, 2015). No en vano, entre los fines y objetivos expresados en 
los estatutos y reglamentos de la mayoría de OTSAS suelen tener mayor peso las cuestiones de sensibilización, defensa o promoción de un colectivo o situación social, que el ejercicio de la función estrictamente prestadora (Ruíz de la Olabuénaga, op. cit.). Por todo ello se hace necesaria una mirada más transversal y ecológica, entendiendo al sector en un contexto y como un actor que genera respuesta de diversa naturaleza a problemas colectivos en un entorno cambiante (Astley \& Fombrun, 1983).

Otro aspecto necesario para delimitar el objeto de trabajo tiene que ver con la concepción o concepciones en torno a la idea de colaboración de las organizaciones. Hilmmelman (1996) entiende la colaboración como un proceso orientado a la búsqueda de beneficio mutuo, con una dimensión organizacional interna y externa, concretado en el intercambio de información, el desarrollo conjunto de actividades o el uso compartido de recursos. Para Iglesias \& Carreras (2013) existe una diferencia entre la colaboración táctica, en la que la relación entre organizaciones no necesita ser intensa y se concentra con frecuencia en espacios de coordinación predominantemente formal; y la colaboración orientada a la creación de valor estratégico, que implica una relación más intensa de las partes y al mismo tiempo obliga a la toma de decisiones relacionadas con factores como la inversión de tiempo y recursos, las cuestiones de liderazgo...

Otra diferenciación es la establecida entre colaboración y partnership (asociación), para Agranoff y McGuire (2003) la primera forma implica una cierta jerarquía ya que las partes comparten objetivos comunes, mientras que la asociación tiene un carácter más mutualista. Iglesias y Carreras (2013) diferencian tres tipos de colaboración entre organizaciones del tercer sector: 1) Las alianzas estratégicas, construidas en relación a un objetivo o logro de interés para todos los integrantes del proceso de colaboración, estando el compromiso temporal y el desarrollo de tareas vinculado a la consecución del mismo; este tipo de colaboración es también frecuente entre organizaciones de ámbitos muy diversos (entidades sociales, administraciones, empresas...). 2) Las integraciones institucionales, que pueden dar como resultado la unificación en una de dos o más entidades o la fusión de distintas entidades en una nueva organización. 3) La creación de redes o plataformas, mediante las que entidades que comparten intereses deciden voluntariamente generar una estructura de cooperación con vocación de permanencia en la que ponen en común conocimientos, experiencia, recursos y en la que puedan unificar objetivos y estrategias de acción. Formalmente estas redes se apoyan en lógicas de reciprocidad (Waddell, 2011).

Las expectativas respecto a los procesos de colaboración son un último aspecto incidente. Para Nieto et al (2013) las entidades del tercer sector están vocacionadas a colaborar por razón de su naturaleza, a ello se unen aspectos contextuales y estratégicos que hacen que, en un contexto global, la supervivencia de cualquier entidad del tercer sector sea imposible sin una estrategia de colaboración (explícita o no). Esta evidencia convive al tiempo con una paradoja, el proceso de constitución del TSAS en nuestro país se ha caracterizado por un desarrollo aluvional, en el que una buena parte del tejido de entidades se ha construido en un periodo espacial y temporal próximo, pero orientado a la búsqueda de respuestas a demandas y problemáticas diferenciadas. Este hecho, sobre el que nos detendremos más adelante, ha impuesto una cierta cultura particularista muy presente aún en el sector (Jaraíz, 2015), que 
con frecuencia añade reticencias internas en las propias entidades que dificultan las dinámicas de colaboración y que se concentra en diferenciaciones en base a aspectos: como el nivel y las funciones de los cuadros profesionales respecto al voluntariado, o las diferencias de capacidad y rol entre grandes y pequeñas organizaciones (Cabra de Luna \& De Lorenzo, 2005).

Como cierre de este apartado podemos decir que los procesos de colaboración de las OTSAS han de ser situados en un contexto explícito. Se desarrollan condicionados por una estructura de capitalismo del bienestar, en torno a la que el tercer sector ha asumido un rol colaborador de diferente naturaleza según el espacio territorial y el momento histórico. La evolución de este contexto macro político ha marcado las propias estrategias de colaboración del sector.

\section{Metodología}

El estudio de las relaciones de colaboración ha sido uno de los cuatro aspectos abordados en el Proyecto PECOTSAS ${ }^{3}$. La estrategia metodológica desarrollada se ha adaptado a los objetivos planteados para cada uno de ellos. En la primera fase de la investigación se describieron las dinámicas de colaboración recurriendo al estudio de fuentes secundarias, principalmente: documentos generados por las OTSAS, contenido corporativo de las redes de colaboración más significativas, contenido en páginas web y análisis bibliográfico. Estas herramientas nos han permitido un relato longitudinal (Caïs, Folguera \& Formoso, 2004) que nos ha servido de guía para la presentación de resultados.

En la segunda fase abordamos el análisis de las percepciones de los diferentes actores. Para ello recurrimos a la búsqueda de información de fuentes primarias mediante entrevistas en profundidad y grupos focales. Para la aplicación de ambas técnicas utilizamos criterios de muestro cualificado (Coller, 2000) vinculados a los siete casos autonómicos de estudio seleccionados: Andalucía, Cataluña, País Vasco, Castilla y León, Comunidad de Madrid, Comunidad de Murcia y Comunidad Valenciana. La selección de comunidades autónoma fue realizada por el equipo de investigación en base a criterios de representatividad de diferentes realidades y contextos.

Las entrevistas en profundidad se hicieron entre los meses de abril y septiembre de 2019. Se realizaron un total de 48 entrevistas en profundidad, utilizando un único guion que contenía preguntas relativas a cada uno de los cuatro aspectos objeto de análisis. Detallamos a continuación el cuadro de entrevistas y los códigos que servirán de referencia:

3. Son cuatro los ámbitos de investigación contemplados en el proyecto: Los aspectos socio-históricos, la relación con las administraciones, las similitudes y diferencias de conjunto entre comunidades autónoma y las dinámicas de colaboración interna y externa. 
Tabla 1. Distribución de las Entrevistas en Profundidad (EP)

\begin{tabular}{|c|c|c|c|}
\hline Comunidad & Informantes & №. Entrev. & Códigos \\
\hline \multirow{2}{*}{ Andalucía } & Internos al sector & 4 & EP 01-04 \\
\hline & Externos y grupos de interés & 2 & EP 05-06 \\
\hline \multirow{2}{*}{ Castilla - León } & Internos al sector & 3 & EP 33-35 \\
\hline & Externos y grupos de interés & 3 & EP 36-38 \\
\hline \multirow{2}{*}{ Cataluña } & Internos al sector & 5 & EP $25-29$ \\
\hline & Externos y grupos de interés & 3 & EP 30-32 \\
\hline \multirow{2}{*}{ Comunidad de Madrid } & Internos al sector & 3 & EP 07-09 \\
\hline & Externos y grupos de interés & 6 & EP 10-15 \\
\hline \multirow{2}{*}{$\begin{array}{l}\text { Comunidad } \\
\text { Valenciana }\end{array}$} & Internos al sector & 3 & EP 39-41 \\
\hline & Externos y grupos de interés & 2 & EP $42-43$ \\
\hline \multirow{2}{*}{ País Vasco } & Internos al sector & 5 & EP 16-20 \\
\hline & Expertos y grupos de interés & 4 & EP 21-24 \\
\hline \multirow{2}{*}{ Región de Murcia } & Internos al sector & 3 & EP 44-46 \\
\hline & Expertos y grupos de interés & 2 & EP 47-48 \\
\hline
\end{tabular}

Fuente: Elaboración propia.

Los diferentes informes de entrevistas por comunidades autónomas fueron elaborados entre junio 2019 y marzo de 2020. Tras el contraste de los mismos se construyó el guion de los grupos focales, orientados a aspectos sobre los que nos parecían de interés su profundización. El hecho de realizar esta parte del trabajo de campo en etapa de pandemia nos hizo reformular en parte el diseño inicial: por un lado las sesiones de los grupos focales se tuvieron que realizar en formato On Line, al tiempo que decidimos incorporar una pregunta sobre el impacto de la COVID-19 en la situación del tercer sector. Se realizaron siete grupos focales con voluntariado de las entidades y otros siete con cuadros intermedios entre los meses de abril y mayo de 2021. Con el resultado de los mismos se actualizaron los informes autonómicos en julio de 2021. Presentamos el cuadro de grupos y los códigos de referencia: 
Tabla 2. Distribución de los Grupos Focales (GF)

\begin{tabular}{|c|c|c|c|}
\hline Comunidad & Grupo Focal & №. Participantes & Código \\
\hline \multirow{2}{*}{ Andalucía } & Mandos Intermedios & 4 & GF03 \\
\hline & Voluntariado & 7 & GFO5 \\
\hline \multirow{2}{*}{ Castilla-León } & Mandos Intermedios & 6 & GFO7 \\
\hline & Voluntariado & 5 & GFO2 \\
\hline \multirow{2}{*}{ Cataluña } & Mandos Intermedios & 4 & GF10 \\
\hline & Voluntariado & 5 & GF06 \\
\hline \multirow{2}{*}{ Comunidad de Madrid } & Mandos Intermedios & 6 & GFO4 \\
\hline & Voluntariado & 5 & GF01 \\
\hline \multirow{2}{*}{ Comunidad Valenciana } & Mandos Intermedios & 6 & GF08 \\
\hline & Voluntariado & 5 & GF09 \\
\hline \multirow{2}{*}{ País Vasco } & Mandos Intermedios & 6 & GF13 \\
\hline & Voluntariado & 6 & GF14 \\
\hline \multirow{2}{*}{ Región de Murcia } & Mandos Intermedios & 6 & GF11 \\
\hline & Voluntariado & 6 & GF12 \\
\hline
\end{tabular}

Fuente: Elaboración propia.

Finalmente, los investigadores que hemos trabajado sobre el aspecto de las relaciones de colaboración hemos recurrido a la codificación y tratamiento de discursos y percepciones con el apoyo de ATLAS ti. La codificación integrada se ha agrupado en dos categorías (relaciones internas al sector y relaciones con otros actores). La presentación de los resultados que hacemos a continuación utiliza las etiquetas principales surgidas en el análisis de discurso para cada categoría.

\section{Resultados}

\subsection{Relaciones internas de las OTSAS. De la diferenciación a la lógica federal}

El proceso de reticularización del sector, que ahora describiremos, es coherente y adquiere su encaje más preciso como relato de conjunto, lo que no implica la negación de la importancia de 
los aspectos autonómicos y locales. Tampoco el hecho de que diferentes comunidades puedan desarrollar un esquema identitario propio, al revés, podríamos decir que la estructura relacional del sector en España se ha construido sobre una cultura federal, lo que pone ya en primer plano la importancia de las dinámicas de colaboración y la permeabilidad entre los procesos locales y de conjunto. Como tendencia podría decirse que los elementos diferenciales de cada territorio, más marcados en los primeros tiempos, han ido diluyéndose para dar lugar a una creciente dinámica de "acercamiento y confluencia" (EP8).

En la secuencia de construcción de la estructura de colaboración pueden identificarse tres momentos o fases. En la primera etapa, el momento previo y la transición a la democracia, aparecen los elementos germinales marcados por un contexto de alta participación cívica, significativa en ámbitos como la discapacidad (Díaz, 2020) o las personas mayores (Amezcua \& Sotomayor, 2021). Estos aspectos inciden en el surgimiento de las primeras organizaciones en las diferentes comunidades, muy conectados lógicamente con factores contextuales e históricos. Los siguientes periodos transcurren ya bajo un sustrato social, político y económico de Estado democrático del bienestar. En este momento se producirá la expansión diversificada del sector (segunda etapa), que llevará a la consolidación de un entramado de redes de segundo nivel, como principal estrategia de colaboración (diferenciada según ámbitos de acción) e interlocución de las organizaciones. Las mutaciones en el ámbito de las políticas públicas producidas tras la gran crisis de 2008 dan paso a una tercera etapa marcada por la reestructuración cohesionadora del TSAS. Aquí adquieren mayor relevancia las redes de tercer nivel y la formación de una estructura relacional tendente a la homogeneización.

\section{Figura 1. Proceso de reticularización del OTSAS}

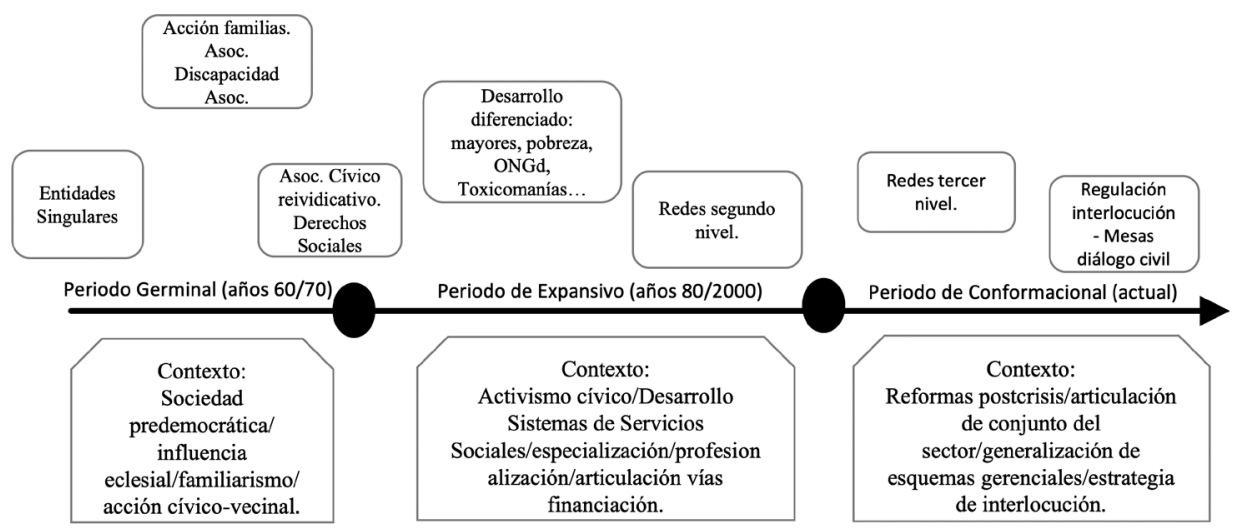

Fuente: Elaboración propia. 


\subsubsection{Periodo germinal (años 60-70)}

Antes de los años 60 existían ya la mayoría de las conocidas como entidades singulares, promovidas principalmente a la sombra de la administración, la Iglesia y las Obras Sociales de las Cajas de Ahorro (Rodríguez \& Montserrat, 1996), este hecho les otorgaba una lógica más institucional que cívica. El impulso modernizador que supuso el desarrollismo predemocráctico va a facilitar el nacimiento de nuevas formas asociativas, más cívicas. Los procesos de colaboración interna de las OTSAS en las comunidades estudiadas están vinculados en origen al nacimiento de estas organizaciones, a partir de los años 60 del pasado siglo.

En la totalidad de comunidades autónomas se identifican dos grandes motores que capitalizan inicialmente al sector: la Iglesia (especialmente el movimiento de parroquias de base) y la movilización de las familias. Con ellos aparece un tercer motor, la colaboración cívico-vecinal de proximidad, que presenta matices en función, más que al contexto autonómico en sí, a elementos como la concentración poblacional (grandes ciudades/zonas rurales) y al grado de desarrollo económico e industrial. Esta cultura colaborativa cívica se expresa en Euskadi mediante el auzolan (trabajo vecinal) y también, por la conexión con el cooperativismo local. Este factor también es reseñable en la Comunidad Valenciana. En Cataluña son referentes los movimientos juveniles de tiempo libre, el movimiento vecinal en los barrios y zonas de asentamiento de población emigrada de otros puntos del país, o la actividad cultural y social de sociedades burguesas y obreras. En Madrid esta vertiente cívica está muy vinculada a los movimientos vecinales. En las zonas rurales de Andalucía o Castilla-León tiene una conexión importante con los espacios de ayuda mutua.

Podríamos decir que en esta primera etapa predemocrática y en la posterior transición, van a ser las relaciones de colaboración las que provoquen la germinación del sector, aprovechando el marco que ofrece la Ley de Asociaciones de 1964. Muchas de las asociaciones dedicada a la discapacidad surgen de la implicación de familias y afectados con el apoyo en ocasiones de asociaciones de cabezas de familia; otras empiezan a organizarse en locales parroquiales, que también sirven, en un primer momento, a la estructuración del propio movimiento vecinal "No había asociación de vecinos que no tuviese local en la parroquia" (EP 21).

En este periodo prima la cooperación natural, poco formalizada y predominantemente centrada en el ámbito local, dada la permeabilidad entre actores de diferente naturaleza. Las propias entidades singulares van a expandirse hacia lo local adoptando estructuras colaborativas (un ejemplo de ello lo encontramos en Cáritas Española que se organiza como una confederación de Cáritas Diocesanas). Pero será en el ámbito de la discapacidad donde, con el impulso de los familiares, comience a implementarse un patrón de colaboración interna más definido y formalizado:

... si miro atrás creo que tuvieron un protagonismo muy importante... empezaron a salir con el tema de la discapacidad, con las asociaciones de familiares... el papel de las familias fue mastodóntico... 


\subsubsection{Periodo expansivo (años 80-2000)}

El segundo momento viene marcado especialmente por el desarrollo de los Sistemas de Servicios Sociales (en adelante SS.SS.) en las diferentes comunidades autónomas: "Hablar de desarrollo de los SS.SS. y del tercer sector es hablar de lo mismo" (GF.3). Las Comunidades Autónomas tratan de construir una red pública de servicios que en la práctica tiene carácter mixto y necesita altos niveles de cooperación con otros actores, especialmente para la prestación de servicios especializados (menores, mayores, personas sin hogar, inmigrantes...).

La expansión de los SS.SS. precisa de tejido cívico, siendo la densidad del mismo diferente en los territorios estudiados. En zonas urbanas y en ciudades pobladas e industrializadas existe ya un razonable nivel implantación del sector (como Madrid o Barcelona, también en Valencia y Sevilla); mientras que en zonas con menor recorrido (como la Andalucía rural y de ciudades medianas) es la propia administración la que contribuye, por necesidad de actores colaboradores, a la promoción desarrollo del sector: "es la administración la que nos dice, venga, créalo y te damos un proyecto (en el ámbito de personas mayores)" (GF.3).

De otro lado, los movimientos de familias y afectados de carácter reivindicativo, o entidades y grupos informales de ámbito vecinal, terminarán articulándose como OTSAS combinando reivindicación y prestación de servicios. Este hecho llevará consigo también la creación de espacios de colaboración interorganizacional en forma de redes o plataformas. Un ejemplo de ello lo encontramos en el ámbito de las drogodependencias con el movimiento Madres Contra la Droga, que arraiga en localidades y barrios de lugares como Galicia, Madrid o Sevilla a finales de los años 80 y que sirve de base para la expansión de asociaciones de lucha contra la droga. En el caso de Andalucía estas organizaciones se agrupan en 1991 en torno a una red de colaboración temática, la Federación ENLACE. Será en este marco de colaboración donde estas OTSAS de carácter local impulsen el desarrollo de programas (prevención en barrios, sensibilización...).

Frecuentemente, la dinámica de expansión de las entidades y la generación de redes de colaboración aparecen como procesos casi simultáneos. En esta expansión empieza también a jugar un papel importante la comentada cultura federal del sector que hace que muchas experiencias producidas en Euskadi, Cataluña o Madrid se transfieren y reeditan con cierta rapidez en otros lugares contribuyendo así a una convergencia de abajo-arriba.

El análisis de la secuencia de constitución de estas redes en este periodo permite apreciar el proceso de especialización y diferenciación creciente del sector. Este fenómeno es especialmente reseñable en el ámbito de la salud y la discapacidad, que es el que dispone de más estructuras de colaboración formalizadas (hemos podido contabilizar 13 redes o plataformas de segundo nivel de ámbito estatal). En otros ámbitos como la lucha contra la pobreza o la cooperación al desarrollo, las redes están más concentradas. Finalmente, en esta etapa se crean también algunas redes de colaboración de carácter transversal como la Plataforma del Voluntariado (1986). Este tipo de espacios ayuda la conexión de organizaciones que operan en diferentes ámbitos específicos, anticipando en cierto modo el posterior surgimiento de entidades de tercer nivel. 
En relación a la naturaleza de los espacios de colaboración la inmensa mayoría son de carácter formalizado. Son redes legalmente constituidas, tendentes a la profesionalización. Las dinámicas de diversificación y formalización de las estructuras de colaboración interna aparecen influidas por las necesidades de las entidades, pero también de las propias administraciones, que les demandan una interlocución más unificada y racionalizadora "necesitamos (la admón.) un ecosistema colaborativo eficiente" (EP37).

En cuanto a las funcionalidades de las dinámicas de colaboración, la principal formulación se concentra en la incidencia política y social respecto a una problemática o colectivo "sobre todo para realmente ser una herramienta eficaz en todo lo relacionado con lo que significa la incidencia política" (GF.8), elemento conectado con la búsqueda de unas condiciones de financiación para la prestación de servicios "no cabe duda de que nuestra relación (entre entidades) está en función a la relación con la administración y con las subvenciones (para el ámbito)" (EP. 6).

Sin embargo, tanto en los relatos de informantes como en la consulta a los elementos corporativos de las redes formales (planes estratégicos, estatutos, webs), es apreciable la evolución hacia una diversificación de contenidos y funciones. Aquí pueden apreciarse otras cuatro áreas de contenido que complementan a las señaladas: 1) La producción de información para la defensa de derechos y la visibilización social; mediante la elaboración de informes, el desarrollo de labores de observatorio, etc. 2) La generación de espacios de transferencia de conocimiento entre entidades a través de creación de grupos de trabajo, oferta formativa común, etc. 3) La promoción de la participación cívica en relación al ámbito de acción de las entidades, a través de mecanismos de fomento y captación de voluntariado, donantes... 4) La regulación de las relaciones, especialmente de las relaciones laborales (convenidos colectivos...) en aquellos espacios con mayores grados de profesionalización.

El grado de colaboración de las organizaciones es un último aspecto a destacar en esta expansión del segundo nivel. Siguiendo el esquema de Carreras e Iglesias (2013), se aprecia la existencia de dos procesos diferenciados. El primero de ellos ha sido el de constitución de redes o plataformas, en las que han primado criterios de reciprocidad. El segundo, menos numeroso pero no marginal, ha sido la integración institucional, de organizaciones pequeñas en una estructura mayor.

Finalmente en este periodo aparecen también algunos espacios de colaboración híbrida, situados a medio camino entre el segundo y el tercer nivel. El Comité Español de Representación de Personas con Discapacidad-CERMI (1997) es un claro ejemplo de ello, como estructura de colaboración que aglutina a 13 redes estatales del ámbito de la discapacidad, así como a los distintos comités autonómicos.

\subsubsection{Periodo conformacional (momento actual)}

La larga crisis que se inicia en 2008 desencadena un nuevo cambio en las OTSAS que tendrá un efecto directo en el desarrollo de su estrategia de colaboración. La dinámica de recorte y el reenfoque de las políticas sociales, sustituyendo programas de inclusión por iniciativas de emergencia social, hace que resulte difícil la supervivencia de una buena parte del sector. Al 
mismo tiempo, este contexto facilita la expansión de entidades lucrativas con capacidad para competir, en escenarios de reducción de costo, por aquellos servicios que tenían alguna capacidad de generación de beneficio (servicios residenciales...).

La merma de entidades sociales en este periodo es puesta de manifiesto en diferentes informes (Fundación Luís Vives, 2012. POAS, 2019). En otros estudios de ámbito local (Jaraíz \& González-Portillo, 2021) hemos estimado que en Andalucía desaparecen, en un periodo de cinco años, en torno a una de cada cinco entidades. Con diferente intensidad esta percepción de reconversión es común en las comunidades estudiadas, "la crisis ha zarandeado al sector... ha puesto cada cosa en su sitio" (EP.5), salvo Euskadi, única comunidad donde los informantes manifiestan que el sector ha presentado buena capacidad de resistencia.

El comentado zarandeo generado por la crisis obliga a revisar el sustrato relacional, "sin perder lo que hacemos de defensa de nuestro ámbito (en las entidades de segundo nivel) necesitábamos defender al sector social y sus problemas comunes" (GF.11). La formación del nuevo momento va a venir marcada por dos experiencias previas: de un lado, por la estrategia seguida por las entidades del ámbito de la discapacidad que fueron capaces de aglutinar al conjunto de redes y plataformas en torno al CERMI; de otro, por la experiencia de la Taula d'entitats del Tercer Sector Social de Catalunya (2003). Tras un periodo de reflexión previo entre entidades singulares, redes y plataformas de segundo nivel de ámbito estatal ${ }^{4}$, en 2012 se crea la Plataforma del Tercer Sector. Esta iniciativa incentiva en los años siguientes la puesta en marcha de plataformas o mesas autonómicas (denominaciones similares), vinculadas a la Plataforma estatal a través de un Comité de Coordinación Territorial.

En general existe una valoración de conjunto de que estas mesas o plataformas de tercer nivel han ejercido una influencia positiva y han reactivado la capacidad relacional del sector "yo creo que representamos al tercer sector más significativo...ahí todos hemos puesto para ser más cordiales entre nosotros" (EP. 3). El principal elemento interno percibido es el potencial para reducir la atomización en la que estaba inmerso el sector: el mayor valor es "el haberse juntado" (EP.29).

En la mayoría de comunidades esta colaboración de tercer nivel se ha concentrado esencialmente en el fortalecimiento de las labores de interlocución y representación del sector ante la administración y ante otros actores (económicos): "a más fuerza, más incidencia política... (somos como) la patronal del sector" (EP.29). El resto de desarrollos es aún desigual entre territorios y parece quedar camino por delante, especialmente en lo relativo a la capacidad de incidencia social "hay que crear (aún) un marco de referencia, de discurso y de relato del sector ante la sociedad" (EP.3). La experiencia de la Taula, la primera de las redes autonómicas, es en este sentido un elemento de referencia por su mayor desarrollo y nivel de visibilización para el conjunto de la sociedad, "han logrado (que el tercer sector sea) un concepto que ya forma parte, digamos, del lenguaje habitual de los medios, del ámbito público, del político..." (EP.32). Diferentes informantes resaltan el valor de esta colaboración en lo relativo a la denuncia y defensa de derechos sociales.

4. En 2010 se convocará la I Convención Nacional del Tercer Sector. 
Finalmente, un factor conectado a la definición de la política de colaboración de tercer nivel es la aparición de las Mesas de Diálogo Civil como estructura formalizada y regulada de relación con las administraciones en diferentes regiones. Este modelo de coordinación abierta administración-tercer sector empieza a desarrollarse en 2012 en Euskadi ${ }^{5}$, pero pronto se convertirá en una reivindicación de las OTSAS en las diferentes autonomías, demanda que será canalizada a través de sus redes de tercer nivel. Como muestra la Tabla 3 , el movimiento pone de manifiesto la comentada permeabilidad entre experiencias autonómicas, después de la iniciativa de Euskadi, otras cinco autonomías han adoptado este modelo de relación OTSAS-administración y en la mayoría de territorios es hoy un asunto de debate vivo.

Tabla 3. Entidades de Tercer Nivel/Mesas de Diálogo Civil

\begin{tabular}{l|l|l} 
Año & Plataformas o Mesas & \\
\hline 2004 & Catalunya & Euskadi \\
\hline 2012 & $\begin{array}{l}\text { Estatal } \\
\text { Extremadura }\end{array}$ & \\
\hline 2013 & Andalucía & \\
\hline 2016 & $\begin{array}{l}\text { La Rioja } \\
\text { Comunidad Valenciana } \\
\text { Asturias }\end{array}$ & Castilla La Mancha Civil \\
\hline 2017 & Comunidad de Madrid & Andalucía \\
\hline 2018 & Canarias & Extremadura \\
\hline 2019 & & Illes Balears \\
\hline 2020 & & Comunidad de Madrid \\
\hline 2021 & Castilla-León & Castilla La Mancha \\
\hline
\end{tabular}

Fuente: Elaboración propia.

Esta última etapa es la que ha aportado más energías homogeneizadoras al sector y con ello a la definición de sus dinámicas de colaboración interna. Este aspecto, valorado como positivo de forma general, es mirado de modo crítico también por una parte de informantes, especialmente voluntariado y dirigentes de entidades que apuestan por una cultura de colaboración más amplia: "todavía tenemos mucho terreno, no solamente normativo, sino de pensamiento... hay aspectos importantes donde no hay una reflexión común de tercer sector..." (EP.3). A este tipo de fragilidades hay que unir las aportadas en otros trabajos (POAS, 2015) en torno a las dificultades de las pequeñas entidades, generalmente de ámbito local, para tomar parte en estos procesos por dificultades como: el acceso a la información o la escasez de recursos técnicos y económicos.

\section{La Diputación Foral de Vizcaya venía funcionado con este método con anterioridad.}




\subsection{Relaciones con otros actores sociales}

\subsubsection{Vínculos generales con los movimientos sociales e incidencia política}

Respecto a la vinculación de las OTSAS en espacios reivindicativos con diferentes movimientos sociales (sindicales, vecinales...) son dos los aspectos que concentran mayor peso: Una parte de las entidades surgieron como respuesta a procesos de reivindicación de derechos, en un contexto de movilización social, este es el caso de un buen número de organizaciones en ámbitos como la discapacidad, las drogodependencias o la cooperación al desarrollo, por citar algunos. De otro lado, persiste la idea de que el sector tiene una función reivindicativa: "Están esas dos facetas del tercer sector, reivindicación y entidad prestadora de servicios” (GF 9).

Sin embargo, esta doble vinculación es percibida como un factor crítico y no fácil de manejar en la práctica:

Las entidades que mantienen incidencia política han sido muy relevantes, pero también la vida diaria para ellas es muy dura... La incidencia política acaba perdiendo peso, porque, es decir, al final la entidad centra el 90\% de su energía o el conjunto de sus energías a prestar un buen servicio porque además me inspeccionan, me supervisan...

Por ello la posición relacional predominante respecto a la implicación en espacios de movilización con otros actores sociales es principalmente mediante conexiones puntuales y no generalizables y en espacios de colaboración táctica en los que se comparte un interés específico. El ámbito en el que se percibe una mayor conexión es probablemente el de las entidades de cooperación al desarrollo, donde el sector ha sido muy activo en campañas como 0,7 ya, 0 Pobreza 0: "Las manifestaciones de Pobreza Cero han sido emblemáticas también muchas de ellas" (EP.42). Aunque la colaboración en procesos de movilización social es bastante desigual entre organizaciones, no es residual en su conjunto, y está presente en diferentes momentos, actualmente en movilizaciones en torno a cuestiones como la garantía de rentas, o el desarrollo de la ley de dependencia... Sí se observa aquí una posición más abierta a la participación en estas dinámicas de colaboración en aquella parte del sector surgida en un contexto de defensa de un derecho.

En gran medida el sector se siente más cómodo generando su propia movilización vinculada a mejoras concretas, visibles y relacionadas a su ámbito de acción. Respecto a la participación en espacios de derecho social más amplio existe un cierto temor a la instrumentalización por otros actores "estamos en un terreno que para mí es política, y la política hoy en día está jugando en contra de las ONG'S" (GF. 5). El modelo ideal de movilización que aparece en las entrevistas y grupos se concentra en la movilización propia, tomando como referencia las realizadas en el ámbito de la discapacidad "no tienen ningún problema si el consejero se resiste, a poner 200 personas con silla de ruedas en la plaza". (EP. 36).

Por otro lado existe también una idea sólida de que el sector aporta un elemento transversal de clara movilización cívica: el voluntariado, como virtud cívica máxima en relación a su 
naturaleza non profit (Marbán, 2001). Esta concepción general tiene un arraigo especial en comunidades como Cataluña, donde es visto por algunos informantes como un elemento constitutivo de la propia cultura y de la asunción (liberal) de las capacidades de la sociedad para organizarse en búsqueda de soluciones propias.

La mirada respecto a la posición de las OTSAS desde el lado de los movimientos sociales es esencialmente crítica, aunque con diferentes matices. En el lado más ácido es observado como un actor "despolitizado (cuyo objetivo es) dulcificar la extrema pobreza" (EP.18). Otras visiones matizan y ponen el foco en los aspectos diferenciales de las OTSAS respecto a otros espacios cívicos "tienen una manera de articularse distinta... un discurso diferente en términos de modelo de sociedad" (EP.16). A pesar de ello el proceso de pérdida de espacio reivindicativo es un elemento recurrente, no solo por la intensidad, también por las formas, frecuentemente con mensajes muy genéricos y formalizados:

(al) tercer sector en el tema reivindicativo... se está quedando con poco espacio, con muy poco espacio...Yo creo que casi con un espacio simbólico, más bien de declaración que de reivindicación...

\subsubsection{Vínculos particulares}

Sobre las relaciones con actores sociales concretos podría decirse que el principal elemento que condiciona la cultura colaborativa del sector es la búsqueda de un espacio propio. Esta idea, que ha ido tomando peso a lo largo del tiempo, se convierte en factor condicionador de las relaciones de colaboración externa, intensificándose tras la crisis de 2008. Todo ello hace que exista una percepción prevalente en el conjunto de informantes de que, con el transcurso de los años, las relaciones con otros actores sociales, como por ejemplo con los movimientos vecinales, se hayan ido debilitando.

En la conexión con lo comunitario es donde aparece una posición autocrítica más marcada "nos hace falta tener sociedad de verdad detrás" (EP11). La tendencia a la especialización en la prestación de servicios ha supuesto en muchos casos el distanciamiento respecto los ámbitos de proximidad. Diferentes discursos señalan la pérdida de espacios de acción común: "las reuniones periódicas con esas asociaciones... para ver las necesidades básicas aquí (ahora) no se producen, por lo menos yo en esta zona... simplemente es, yo saco una convocatoria, tú me mandas un proyecto..."(GF.5) y apuntan que la recuperación del espacio colectivo de proximidad supone un reto y un elemento de renovación para el sector "hablamos del tema de los barrios, de marginación... yo creo que queda mucho todavía (continua hablando de aspectos locales)". (EP.3). Este tipo de dinámica de colaboración local y su potencial de innovación en formas de procomún están presentes sin embargo en algunos entornos:

la dispersión y la ruralidad actualmente están propiciando experiencias innovadoras muy pegadas al terreno y muy vinculadas a los movimientos sociales de soberanía alimentaria, de cercanía a la tierra... 
Las organizaciones de economía social son otro de los actores recurrentes en relación a la colaboración externa. En la conexión entre ambos convive una dualidad de percepciones. Por un lado las OTSAS han optado, como ya se ha argumentado, por la definición de un espacio propio y diferenciado, tanto por su identidad no lucrativa, como por la idea de que desde el propio ámbito de la economía social las OTS son otra cosa "tanto el cooperativismo, como las asociaciones laborales han sido bastante reacios a reconocer al tercer sector como parte de la economía social...Aquí ha habido siempre bastante recelo" (EP.24). Esta posición convive con la apuesta de parte del sector por soluciones de economía social (fomento de empresas de inserción, centros especiales de empleo...). Estas soluciones son vistas, más que como elemento institucional propio, como una solución autónoma para una parte de los beneficiarios de los programas (Cea, 2021). La visión desde las organizaciones de economía social de esta relación es también crítica, aunque existen diferencias entre entidades que comparten con las OTSAS el vínculo con lo social respecto entidades de economía más abierta de lógica más cooperativa. En este espacio se reconoce la dificultad relacional del tercer sector, especialmente respecto a los aspectos laborales: "las ONGs dan menos importancia a cosas como las cláusulas sociales y a los aspectos salariales y de garantía de condiciones de trabajo, esto es una desventaja para nosotros" (EP.4). La economía social observa al sector como una especie de marca blanca que en ocasiones genera desventaja competitiva.

El ámbito sindical es el que concentra una percepción más crítica respecto al sector; en ello influye también el momento relacional específico y el reparto de espacios de poder en torno a la visibilización y capacidad de concertación política: "los sindicatos no nos quieren ver en ámbitos laborales, porque es su ámbito... ellos tienen menos problema para entrar en ámbitos sociales.... ahí hay una relación complicada". (EP.23). En distintas comunidades las OTSAS perciben una resistencia sindical a compartir este espacio de interlocución, especialmente en los procesos de constitución de las Mesas de Diálogo Civil "nosotros pedimos esta mesa de diálogo social, los sindicatos dicen con estos señores no nos sentamos...Ojo, que con ellos nos sentamos también, pero vamos, no, no" (EP.34).

El último de los espacios relacionales se concentra en el mundo empresarial, respecto al que aparece una nueva dualidad. Se observa el potencial del tejido como soporte financiador del sector, especialmente en relación a los aspectos de responsabilidad social corporativa; pero de otro lado se percibe la amenaza competitiva de la mercantilización de los servicios sociales y la existencia de un subsector social vinculado a lógicas de marketing corporativo "(el sector mercantil) no solo compite con bajos precios... (también) con su lado social...por razones de convencimiento, marketing o lo que sea" (EP.12).

\section{Discusión y conclusiones}

Respecto al primero de los propósitos de la investigación (análisis de las lógicas relacionales y los efectos buscados en los procesos de colaboración del sector), los resultados de este trabajo ponen de manifiesto cómo las dinámicas de colaboración del sector han sido uno de 
los elementos críticos en su configuración y desarrollo. Las OTSAS han evolucionado en un marco de pluralismo del bienestar en el que, con procesos de expansión y retracción, el Estado ha ejercido de impulsor principal de su capacidad de acción, habilitando espacio y recursos (Alexander, 2000. Cabra de Luna, 2016. Rodríguez Cabrero, 2020). Pero el sector también ha evolucionado y se ha conformado en base a la construcción de juegos de interdependencia entre sus organizaciones.

El proceso de construcción de redes llevado a cabo por el sector se apoya en su origen en una doble base: reivindicativa-cooperativa, en cuanto a que las principales iniciativas de colaboración entre entidades se hacen para la inclusión de un problema o fenómeno social en la agenda de política pública; y la articulación de respuestas a necesidades conectadas con ello mediante la prestación de servicios. En ambas dimensiones las relaciones buscan una respuesta, principalmente de la esfera pública, también de la sociedad en su conjunto (reconocimiento y visibilización, adhesiones...). Pero al mismo tiempo, la gestión de los procesos de colaboración ha generado resortes internos que contribuyen decisivamente a la cualificación del sector.

Esto es así porque, en línea con otros trabajos (Vélez, 2007), las organizaciones ponen en juego y al mismo tiempo tratan de expandir sus recursos económicos, su acceso al manejo de información y, finalmente, el desarrollo de su capital social. Éste último es un aspecto central y diferencial del aporte del sector respecto a otros actores. En el caso español el principal desarrollo capitalizador ha sido participado por las redes de segundo nivel, el más definido y extenso (no es casualidad que estas redes sean las más visibles para los informantes y, especialmente para el voluntariado).

Un primer elemento capitalizador al que han contribuido se refiere al fomento de la participación ciudadana mediante el voluntariado, todo ello en un contexto de pérdida general de espacios de participación y de reducción de conjunto del capital asociativo en la sociedad española, (FOESSA, 2018). Diferentes redes de colaboración han contribuido al desarrollo de herramientas de captación y orientación, formación... para las personas voluntarias y para sus organizaciones integrantes.

En línea similar, buena parte de estos espacios de colaboración han asentado su legitimación generando un entorno de servicios internos para la gestión del conocimiento, principalmente mediante la creación de herramientas de coordinación, oferta de formación a cuadros dirigentes y profesionales y otros elementos vinculados a la producción y transferencia de conocimiento.

Finalmente, un tercer aporte socialmente capitalizador es el relacionado con la mejora de la calidad, tanto en la gestión organizacional, como en la prestación de servicios, facilitando instrumentos y apoyos para la planificación estratégica e implementación de políticas de calidad en el sector.

La irrupción, con más solidez en el último tiempo, de iniciativas de colaboración de tercer nivel aporta un elemento paradójico. De un lado, los dirigentes y profesionales de OTSAS (éstos en menor medida) valoran su utilidad como impulsoras de conjunto del sector, sin embargo, este proceso ha permeado menos en los niveles intermedios y en el nivel básico (especialmente en el voluntariado). El mayor logro de esta dinámica de colaboración está en la mejora de 
las condiciones de interlocución, una especie de estación de llegada para algunos agentes del sector, pero no para otros, que entienden que estos espacios de colaboración han de facilitar lo que un informante ha llamado la construcción de un "relato del sector". (EP.3)

Persisten dos visiones de distinto recorrido en relación a ese relato a construir. La primera de ellas, tal vez sobre la que más energías se han puesto en este momento del proceso, se concreta en la definición de una posición propia y diferenciada respecto a otros actores, en buena parte por la convicción de que, en sus líneas maestras, las políticas públicas han de priorizar sobre el conjunto los aportes de valor añadido del sector. La segunda, sin negar esa dimensión genuina, aboga por una construcción bottom-up del relato, buscando aproximaciones cualitativas a los problemas objeto de intervención, conectando los elementos de conjunto con los procesos locales (Finn, 2000). En cierto modo, esta reivindicación de las OTSAS respecto a los espacios de colaboración, viene a poner sobre la mesa una cuestión esencial (sobre las que una parte del sector reconoce la debilidad propia y demanda el apoyo de sus estructuras de colaboración y relación interna): la necesidad de posicionar al sector en los espacios de innovación social vinculados a la proximidad.

Existe un desajuste entre una lógica de reticularización marcada por la especialización y la diferenciación de las prácticas (cada entidad en su espacio), y otra que aboga por la construcción de entornos de bien común, en la que las OTSAS han de aprender a generar y gestionarse en contextos relacionales amplios. Este aspecto incide, además, en otros elementos internos, como las dinámicas de profesionalización predominantes en el sector, con frecuencia más decantadas a la especialización que al trabajo comunitario. También, en similar sentido, de las formas de liderazgo institucional.

En este espacio de conexión innovación-proximidad está contenido uno de los mayores elementos de generación de conocimiento, o mejor dicho, de sistematización y transferencia, ya que existe experiencia y know-how en aspectos como: la organización de espacios de bien común y comunidades de práctica (Wenger, 1998; Saz et al, 2016), de formatos de gestión conjunta de servicios entre OTSAS y de economía social (Crespo, 2013), o la implementación de estrategias de participación y modelos de gestión organizacional abierta (Gracia \& Santos, 2012).

Del mismo modo que la implementación de redes de segundo nivel contribuyó a una capitalización diferenciada del sector (en torno a distintos ámbitos de actividad), el desarrollo de espacios de tercer nivel hace que una parte importante de OTSAS adquiera conciencia de las posibilidades de asentar colectivamente modos de hacer y de estar que den solidez al sector. En los discursos se apunta que el desarrollo de esta vía está aún en estado inicial, reconociéndose como un factor clave la profundización sobre la misma.

El segundo aspecto de interés en este trabajo tenía que ver con las diferencias territoriales en los procesos de colaboración entre entidades, para ver en qué medida éstas podían influir en la configuración de modelos diferenciados de tercer sector. Los relatos de los informantes y el trabajo sobre fuentes secundarias ponen de manifiesto la existencia de contextos diferenciados de generación de redes. En este sentido el trabajo identifica tres tipos de elementos incidentes que podemos llamar factores comunes, diferenciados y contextuales. 
En relación a los factores comunes en el conjunto de territorios aparece la influencia de la Iglesia y de las familias como impulsores de las OTSAS, elemento que es visto como incidente en todo el proceso (desde los inicios hasta hoy) y que ha sido tratado en diferentes trabajos (Cabra de Luna \& De Lorenzo, 2005; Díaz, 2020). A ello se une también el vigor asociativo de la transición y los principios de la democracia. Estos elementos han tenido un arraigo diferenciado, más que entre comunidades (que también), entre contextos urbanos industrializados en un extremo y contextos rurales en el otro.

En relación a los factores diferenciados estos tienen un especial peso en los relatos de Cataluña, Euskadi y Comunidad de Madrid. En Cataluña se asocia la intensa visibilización del sector y el peso cívico y político de la Taula, con la tradición asociativa y la percepción del voluntariado como un fenómeno muy extendido y transversal a toda la sociedad. En Euskadi se apunta a la confluencia entre cultura cooperativa y las dinámicas de colaboración local y, con ello, al papel peculiar de la Iglesia en la construcción de las redes de colaboración, donde han participado de su impulso y, al mismo tiempo, han facilitado una capitalización cívica del mismo. En ambas comunidades aparece, en diferente forma, la asunción de la subsidiariedad como criterio de acción política, tanto por los partidos que han ejercido el poder en periodos de tiempo largo (principalmente de corte demócrata-cristiano), como por la propia sociedad civil. Si bien es importante anotar que en Cataluña no aparecen evidencias tan explícitas de su impacto en las lógicas de gobernanza como en Euskadi, donde, en línea con trabajos como los de Arrieta y Sobremonte (2016), los poderes políticos han posibilitado un marco de colaboración comparativamente más sólida que en otros territorios. Los discursos de informantes de la Comunidad de Madrid aluden a dos elementos diferenciales: uno, las sinergias reivindicativas con el movimiento vecinal (este es común en el conjunto de ciudades); dos, el efecto de capitalidad y la capacidad de concentración de redes y lobbies y, con ello, de atracción y transferencia de innovaciones respecto a otros territorios. Este efecto de capitalidad aparece también en Barcelona respecto a Cataluña.

En cuanto a los factores contextuales, son diversos los trabajos que ponen de manifiesto como, en el momento actual, los diferentes regímenes o modelos de bienestar social europeos están confluyendo en sus dinámicas de gobernanza, implementando en todos o la mayoría de sus ámbitos de política social fórmulas de co-producción o co-creación de servicios (Pestoff, et al, 2013; Voorberg, et al, 2015). Este escenario tiende a homogeneizar las estrategias de colaboración sector público-tercer sector, una tendencia que aparece con claridad cuando se analiza el proceso reciente de desarrollo de las estructuras de tercer nivel del sector y, casi simultáneamente, el despliegue de iniciativas de diálogo cívico impulsadas desde las diferentes administraciones autonómicas.

Poniendo en diálogo los tres tipos de factores podemos concluir que existen diferencias territoriales en el desarrollo de las estrategias de colaboración: unas han desarrollado más la cultura cívico-participativa, otras los aspectos de gobernanza y coordinación abierta con el ámbito público y otras el desarrollo de un tejido relacional denso y la formación de grupos de interés. Estas diferencias otorgan identidades específicas a diferentes comunidades, sin embargo, miradas en su conjunto, no conforman modelos de colaboración diferenciados. Esto es 
así por el predominio en el momento actual de dinámicas de homologación influidas, además por el contexto geopolítico global, por la existencia en el caso español de una cultura relacional federal que posibilita un destacable grado de permeabilidad de iniciativas entre territorios.

Contribución de cada autor/a: Autor A: Trabajo de campo, análisis de datos y redacción y revisión final del texto; Autor B: Trabajo de campo, análisis de datos y redacción del texto.

Financiación: El proyecto PECOTSAS (CS02017-84856-P) ha sido financiado por el Ministerio de Economía , Industria y Competitividad en el marco del Plan Nacional de I+D+i.

\section{Bibliografía}

AGRANOFF, R. \& McGUIRE, M. (2003): Collaborative public management. New strategies for local governments, Washington: Georgetown University Press.

ALEXANDER, J. (2000): "Adaptive strategies of nonprofit human service organizations in an era of devolution and new public management", Nonprofit Leadership and Management, 10 (3), 287-303. http://dx.doi.org/10.1002/nml.10305

AMEZCUA-AGUILAR, T. \& SOTOMAYOR-MORALES, E. (2021): "Dinámicas de participación en la vejez: Del asociacionismo tradicional al activismo sin carné", OBETS, Revista de Ciencias Sociales, 16 (1), 35-50, https://doi.org/10.14198/OBETS2021.16.1.02

ARRIETA, F. \& SOBREMONTE, E. (2016): "Elementos para un relato de la gobernanza del Sistema Vasco de Servicios Sociales", Zerbitzuan, Revista de Servicios Sociales, 61, 5-21.

http://dx.doi.org/10.5569/1134-7147.61.01

ASTLEY, W.G. \& FOMBRUN, C.J. (1983): "Collective strategy: Social ecology of organizational environments", Academy of Management. The Academy of Management Review, 8 (4), 576. https://doi.org/10.2307/258259

BEATON, E., MACINDOE, H. \& WANG T. (2021): "Combining Nonprofit Service and Advocacy: Organizational Structures and Hybridity", Nonprofit and Voluntary Sector Quarterly, 50(2), 372-396, https://doi.org/10.1177\%2F0899764020959477

BILLIS, D. (1992): "Planned Change in Voluntary and Government Social Agencies", Administration in Social Work, The Quaterly Journal of Human Services Management, 16 (4), 29-44. https://doi.org/10.1300/J147v16n03_03

CABRA DE LUNA, M.A. \& DE LORENZO, R. (2005): “El tercer sector en España. Ámbito, tamaño y perspectivas", Revista Española del Tercer Sector, 1, 95-134. 
CABRA DE LUNA, M.A. (2016) "Realidad del Tercer Sector en España y crisis del Estado de Bienestar: retos y tendencias", EHQUIDAD, Revista Internacional de Políticas de Bienestar y Trabajo Social, (1), 115-134. https://doi.org/10.15257/ehquidad.2014.0005.

CAÏS, J., FOLGUERA, L. \& FORMOSO, C. (2014): Investigación cualitativa longitudinal, Colección Cuadernos Metodológicos, 52, Madrid: CIS.

CARRERAS, I. \& IGLESIAS, M. (2013): La colaboración efectiva de las ONGs. Alianzas estratégicas y redes, Barcelona: ESADE.

CASADO, D. \& FANTOVA, F. (coord.) (2007): Perfeccionamiento de los Servicios Sociales en España. Informe con ocasión de la ley sobre autonomía y dependencia, Madrid: FOESSA.

CEA, J.C. (2021): “Cooperativismo loco. Trabajo, comunidad e inclusión laboral en salud mental", Cuadernos de Relaciones Laborales, 39 (1), 113-131. http://doi.org/10.5209/crla.76228

COLLER, X. (2000): “Estudio de Casos”, Colección Cuadernos Metodológicos, 30, Madrid: CIS.

CRESPO, T. (2013): “Una nueva relación del Tercer Sector y la economía social”, Cuadernos de Trabajo Social, 26(1), 65-74. https://doi.org/10.5209/rev_CUTS.2013.v26.n1.4075

DIAZ, E. (2020): "El asociacionismo en el ámbito de la discapacidad. Un análisis crítico", Intersticios. Revista sociológica de pensamiento crítico, 12 (2), 29-48.

EVERS, A. \& SVETLIK, I. (Dirs.) (1993): Balancing Pluralism. New Welfare Mixes in Care for the Elderly, Aldershot: Ashgate Publishing Company.

EIKENBERRY, A.M. \& KLUVER, J.D. (2004): "The marketization of the nonprofit sector: Civil society at risk?", Public Administration Review, 64 (2), 132-140.

ESPOSITO, P., BRESCIA, V., FANTAUZZI, C. \& FRONDIZI, R. (2021): “Understanding Social Impact and Value Creation in Hybrid Organizations: The Case of Italian Civil Service", Sustainability, 13(7):4058. https://doi.org/10.3390/su13074058

FINN, D. (2000): "Welfare to Work: The local dimension", Journal of European Social Policy, 10 (1), 43-57. http://dx.doi.org/10.1177/095892870001000103

FUNDACIÓN FOESSA (2018): VIII Informe sobre exclusión y desarrollo social en España, Madrid: FOESSA.

FUNDACIÓN LUIS VIVES (2021): Anuario del Tercer Sector de Acción Social, Madrid. 
GONZÁLEZ-PORTILLO, A. \& JARAÍZ, G. (2017): "Voluntariado y Tercer Sector en Andalucía: revisando el pasado, mirando al futuro". En: Márquez, M.A. et al. (coords.) (2017): La incidencia de las organizaciones de la sociedad civil en las políticas públicas del ámbito local. Estudios sobre la región Autónoma de Andalucía, España y la Ciudad de México, Ed. Universidad Nacional Autónoma de México.

GRACIA, M. \& SANTOS, J. (2021): "Nuevas formas de participación política como elemento central en la construcción de nuevos modelos de ciudadanía: las postconvencionales", Política y Sociedad, 58 (2). https://doi.org/10.5209/poso.68745

HIMMELMAN, A.T. (1996): "On the theory and practice of transformational collaboration: From social service to social justice". In: Huxham, C. (dir.): Creating collaborative advantage, Thousand 0aks: Sage Publications.

JÄGER, U.P. \& SCHRÖER, A. (2014): "Integrated Organizational Identity: A Definition of Hybrid Organizations and a Research Agenda", VOLUNTAS Int. J. Volunt. Nonprofit Organ., 25, 12811306. https://doi.org/10.1007/s11266-013-9386-1

JARAÍZ, G. (2015): "El tercer sector como sociedad civil. Dialógicas y recursividades en un contexto de crisis", Revista Española del Tercer Sector, 30, 99-126.

JARAÍZ, G., GÓNZALEZ-PORTILLO, A. \& HERNÁDEZ LEÓN, E. (2021): “Diagnóstico de las organizaciones del Tercer Sector de Acción Social de Andalucía". En: Construyendo conocimientos para una transformación social inclusiva. Aportes a la agenda de investigación del trabajo social, Valencia: Tirant lo Blanc.

JARILLO, J.C. (1988): "On strategic networks", Strategic Management Journal, 9 (1), 31-41. https://www.jstor.org/stable/2486000

MARBÁN, V., PÉREZ, M. \& RODRÍGUEZ, G. (2020): “Current challenges and prospective analysis of the third sector of social action (TSSA) in Spain", CIRIEC-España, Revista de Economía Pública, Social y Cooperativa, 98, 95-126. https://doi.org/10.7203/CIRIEC-E.98.15385

MARBAN, V. (2001): "Sociedad civil, tercer sector y entidades de acción social en España", RIS. Revista Internacional de Sociología, 30, 169-205. https://doi.org/10.3989/ris.2001.i30.772

MONZÓN, J.L. (2009): “Tercer Sector. Delimitaciones Conceptuales y Situación Ocupacional”, Panorama Social, 9, 38-46.

NIETO, E. (2009): Procesos de Colaboración entre Organizaciones No Lucrativas: Aplicación al Caso de las ONG españolas, Tesis Doctoral, Facultad de CC. Económicas y Empresariales de la UAM.

NIETO, E. \& DE REINA, R. (2013): "La colaboración como valor diferencial del sector no lucrativo: Aplicación al caso de las 0NGs", Revista de Estudios Económicos, 1 (2e época), 44-46. 
NIETO, E., GARCÍA, J., REYNA, R. \& ROBINA, R. (2013): “Los roles de colaboración entre las principales ONG españolas", CIRIEC-España, Revista de Economía Pública, Social y Cooperativa, 79, 87-114.

PESTOFF, V., BRANDSEN, T. \& VERSCHUERE (2013): New Public governance, the third sector and co-production, London: Routledge.

PLATAFORMA DE ONG DE ACCIÓN SOCIAL (2019): Informe de elaboración del Directorio del Tercer Sector de Acción Social, Madrid: POAS.

PLATAFORMA DE ONG DE ACCIÓN SOCIAL (2015): Análisis prospectivo de los retos actuales y futuros del Tercer Sector de Acción Social en España, MADRID: POAS.

RENDUELES, C. (2017): "Las condiciones institucionales de una reconstrucción de los bienes comunes en sociedades mercantilizadas". En: Rebeldías en común. Sobre comunales, nuevos comunes y economías cooperativas, Madrid: Libros en Acción.

RODRÍGUEZ CABRERO, G. (2020): "El tercer sector de acción social en España. Desarrollo reciente y retos de futuro", Anales de Derecho y Discapacidad, 5, 221-240.

RODRÍGUEZ CABRERO, G. \& MONTSERRAT, J. (Dir.) (1996): Las entidades voluntarias en España. Institucionalización, estructura económica y desarrollo asociativo, Madrid: Ministerio de Asuntos Sociales.

RUIZ DE OLABUÉNAGA, J.M. (dir.) (2006): El sector no lucrativo en España. Una visión reciente, Madrid: Fundación BBVA.

SALAMON, L.M. \& ANHEIER H.K. (1997): Defining the Non-Profit Sector: A Cross-National Analysis, Manchester University Press.

SALAMON, L.M. \& ANHEIER, H.K. (2001): "La sociedad civil en una perspectiva comparativa". En: Salamon, L.M., Anheier, H.K., List, R., Toepler, S., Sokolowski, S.W. et al., La sociedad civil global. Las dimensiones del sector no lucrativo, Bilbao: Fundación BBVA.

SAZ-GIL, I., ALMAGUER-KALIXTO, P. \& GÓMEZ-QUINTERO, J.D. (2016): “Capital social y redes sociales: análisis del Tercer Sector en contextos rurales", CIRIEC-España, Revista de Economía Pública, Social y Cooperativa, 86, 123-154.

SECINARO, S., CORVO, L., BRESCIA, V. \& IANNACI, D. (2019): "Hybrid Organizations: A Systematic Review of the Current Literature", Int. Bus. Res, 12, 1-21.

https://doi.org/10.5539/ibr.v12n11p1

VÉLEZ, G. (2007): "Tendencias del tercer sector a partir del análisis de las relaciones de interdependencia", REDES, Revista Hispana para el Análisis de Redes Sociales, 12.

https://doi.org/10.5565/rev/redes.493 
VOORBERG, W.H., BEKKERS, V.J.J.M. \& TUMMERS, L.G. (2015): "A Systematic Review of Co-Creation and Co-Production: Embarking on the social innovation journey", Public Management Review, 17 (9), 1333-1357, https://doi.org/10.1080/14719037.2014.930505

WADDELL, S. (2011): Global Action Networks. Creating Our Future Together, Palgrave Macmillan.

WENGER, E. (1998): Communities of practice: learning, meaning, and identity, Nueva York: Cambridge University Press.

ZURDO, A. (2007): "La dimensión corporativa del tercer sector. Los tipos organizativos del voluntariado", Revista Internacional de Sociología, 47, 117-143. 